\title{
PROGRESS REPORT ON BEAM-BEAM COMPENSATION WITH ELECTRON LENSES IN TEVATRON
}

\author{
V. Shiltsev, Yu.Alexahin, G.Kuznetsov, N. Solyak, X.Zhang, FNAL, Batavia, IL 60510, USA \\ K.Bishofberger, UCLA, Los Angeles, CA 90095, USA, M.Tiunov, BINP, Novosibirsk, Russia,
}

\section{Abstract}

We discuss the original idea of beam-beam compensation (BBC) in Section I, sequence of events in 2001-2002 and use of the Tevatron Electron Beam (TEL) for DC beam removal in Section II, (anti)proton lifetime improvement in Section III, experimental data on the BBC attempts in Section IV and, conclusively, Section V is devoted to discussion on important phenomena, needed improvements and future plans.

\section{ORIGINAL GOALS OF BBC}

The idea of beam-beam compensation in the Tevatron proton-antiproton collider [1] originally assumed installation of a single low-energy high-current DC electron beam device which would create nonlinear space charge force acting on antiprotons and compensating in average electromagnetic forces due head-on collisions with protons in the two collision points. Later, it was realized that because of non-uniform bunch loading scheme in the Tevatron Run II - each beam contains 3 trains of 12 bunches spaced by $396 \mathrm{~ns}$, the trains are separated by $2.6 \mu$ s gaps - antiproton bunch dynamics depends on bunch position in the bunch train, and two electron lens with pulsed and variable currents can be used to compensated bunch-by-bunch differences, e.g. tune variation $[2,3]$.
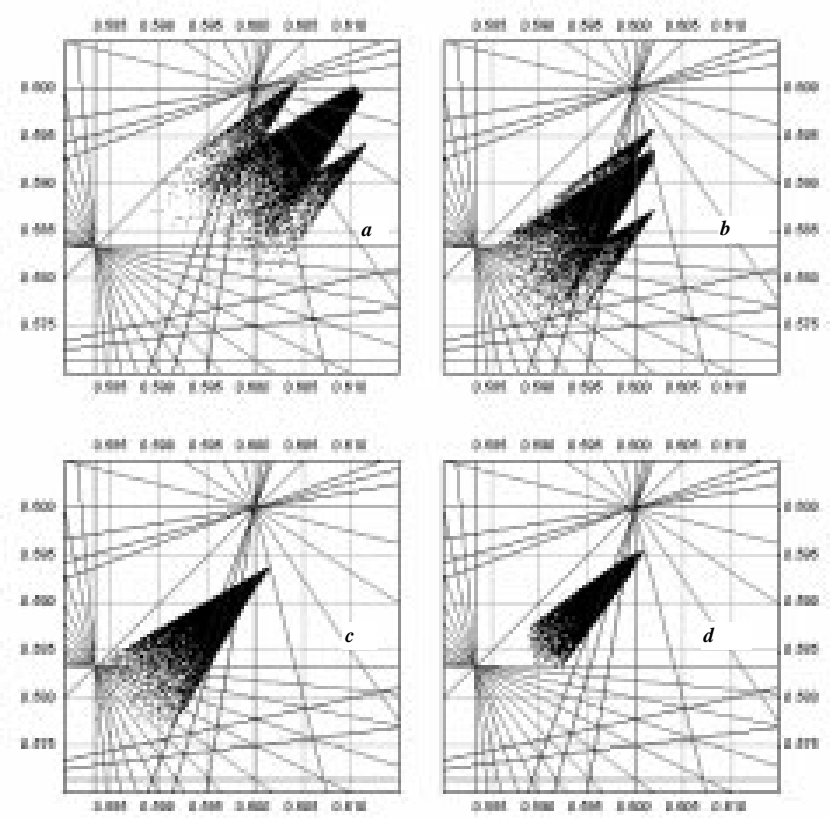

Fig.1: Tevatron tune diagram (a) and various BBC (b,c,d)
Fig.1 shows antiproton tune diagram for design Run II parameters without BBC (Fig.1a), with a single TEL installed at the location with $\beta_{X}>>\beta_{Y}$ and compensating variations of tuneshift in horizontal plane (Fig.1b). If the second linear lens set at a location with $\beta_{Y}>>\beta_{X}$ then antiproton footprint can be reduced as depicted in Fig. 1c, while optimization of electron beam current density profile may result in even further reduction of the antiproton tunespread, Fig.1d. Analytical calculations and numerical tracking $[4,5]$ showed that the BBC should lead to significant improvement of lifetime of some bunches (outliers in Fig.1, bunches \#1 and \#12 in each train) and, thus, should improve integrated luminosity by $5-10 \%$.

That was the ultimate justification for design and construction of the first TEL which started in 1998. In spring 2001 the first TEL has been installed in the Tevatron tunnel and commissioned (see Fig.2).

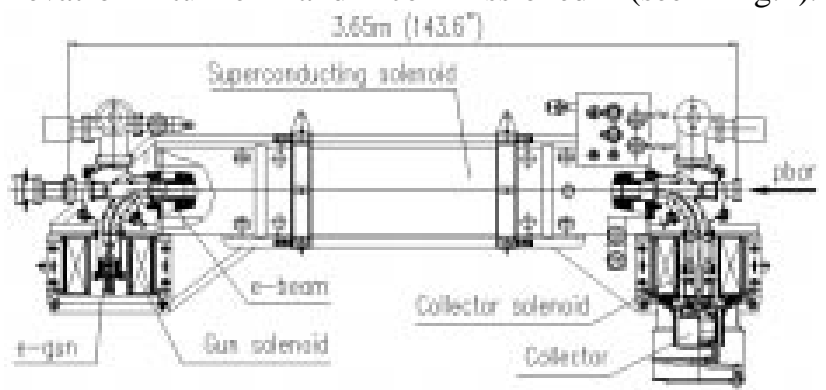

Fig.2: The first Tevatron Electron Lens

Detailed description of magnetic, vacuum and electron beam system of the TEL, its diagnostics and operation can be found in [6], see also references therein.

\section{PROJECT PROGRESS IN 2001-2002}

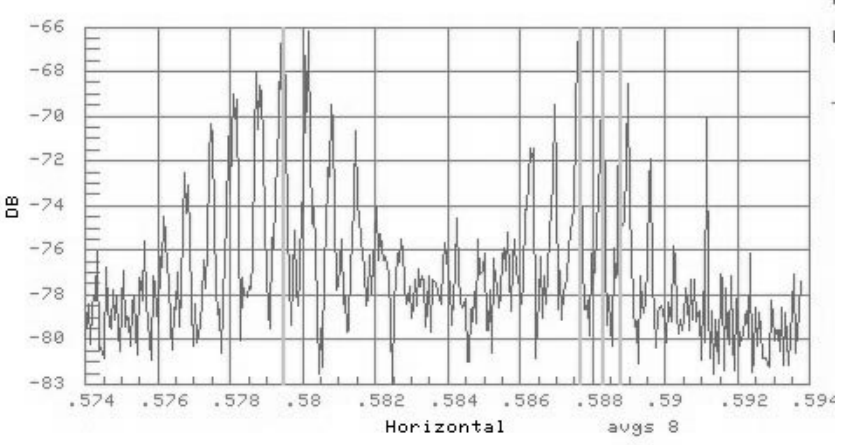

Fig.3: Horizontal tune of $980 \mathrm{GeV}$ protons shifted by TEL

In the first series of beam studies in 2001-2002, we achieved tuneshifts of $980 \mathrm{GeV}$ protons of about $d Q=+0.008$ with $\sim 3$ A of the electron beam current [7]. 
The original $10 \mathrm{kV}$ electron gun generated constant current density distribution in $3.4 \mathrm{~mm}$ diameter beam over $2 \mathrm{~m}$ long interaction region. Schottky detectors in the Tevatron are used to measure the tunes of the proton bunches. During one test of the lens, three proton bunches (without antiprotons) were injected into the Tevatron and ramped to $980 \mathrm{GeV}$, and the observed (fractional) horizontal tune of all three bunches was 0.5795. Then the lens was pulsed in order to interact with only one of the three bunches. The spectra associated with the other two bunches remained unaltered, but the third shifted by 0.0082 to 0.5877 . Figure 3 shows the resulting spectra; the two untouched bunches produced the set of peaks on the left, and only after turning the TEL on did the third bunch produce the set on the right.

The tuneshift dependence on electron current and energy, on electron beam position and timing was found in good agreement with theoretical formula $[1,6]$.

$$
d Q_{x, y}=\mp \frac{\beta_{x, y}}{2 \pi} \cdot \frac{1 \pm \beta_{e}}{\beta_{e}} \cdot \frac{J_{e} L_{e} r_{p}}{e \cdot c \cdot a_{e}^{2} \cdot \gamma_{p}}
$$

The proton lifetime was in the range of 10 hours (some 24 hours at the best). At first, it was not clear what was limiting it - electron beam current/position fluctuations or nonlinear beam-beam effects complicated by inaccurate electron beam alignment with respect to (anti)protons.

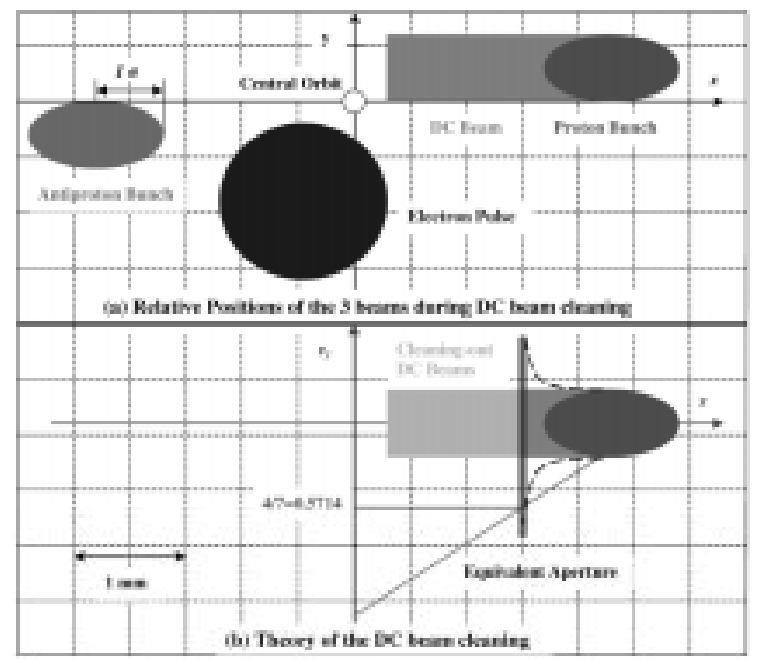

Figure 4: DC beam cleaning: (a) beam positions (b) physics of the abort gap cleaning

In 2002 the TEL was found to be an invaluable instrument for cleaning DC beam in the Tevatron - the application which was not foreseen at the start of the project. The DC beam consists of particles slowly leaking from RF buckets at $980 \mathrm{GeV}$ and circulating all around the ring unsynchronized with RF, thus, present in the abort gap between bunch trains. A few $10^{9}$ particles are enough to cause quench on beam abort. Betatron tunes of Tevatron beams -0.583 in horizontal and 0.575 in vertical plane - are close to $4 / 7^{\text {th }}$ resonance line at 0.5714 . The TEL current is fired in the gaps between bunch trains every $7^{\text {th }}$ turn and thus excite the DC beam particles to very large amplitudes until they are lost - see schematics of transverse positions of three beams and physics of cleaning in Fig.4. Since early 2002, the TEL is being operationally used for the DC beam cleaning in every Tevatron HEP store [8].

\section{LIFETIME IMPROVEMENT}

Our studies in 2001-2002 did show that mis-steering of the electron beam is by far the most important factor affecting the (anti)proton lifetime $\tau=(d N / d t / N)^{-1}$. It can affect $\tau$ even at comparatively small electron currents. Lifetime dependence on the electron current with fixed steering correctors was roughly $\tau \propto 1 / J^{2}$.

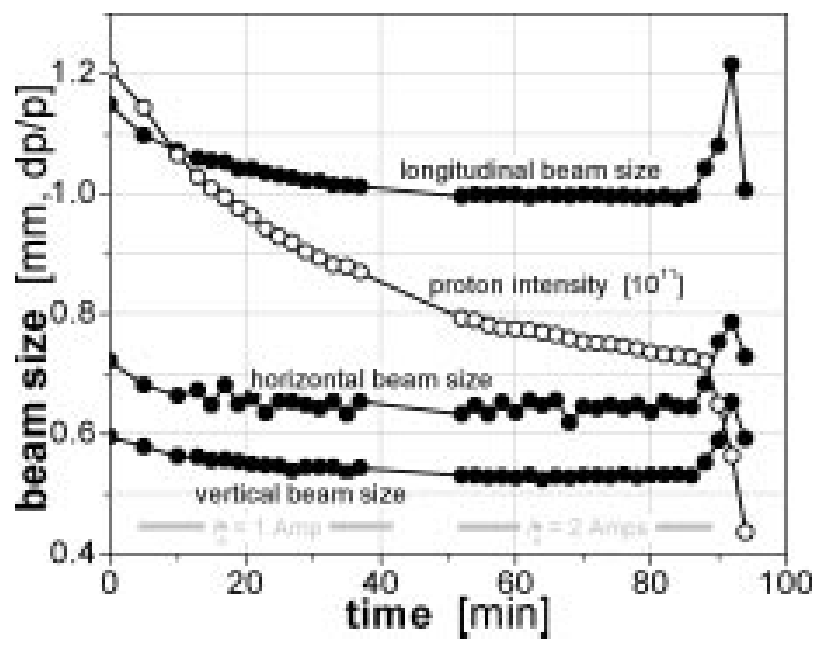

Fig.5: TEL as a "soft collimator"

Eventually, we realized that edges of the electron beam act as a "soft collimator". For example, Figure 5 shows the size of a particular bunch while it was collimated in this manner. One amp of electron-beam current was applied initially. Many particles were quickly lost, decreasing the beam size; however, the loss rate began to level off because the remaining core bunch was stable. To confirm our understanding, the beam current was doubled to two amps, but the beam size was still secure. Also shown is the bunch intensity (open circles) in units of $10^{11}$ particles, and the linear attrition rate indicates that there was a uniform, slow diffusion of particles in phase space, which caused a small amount of continuous losses. At the very end of the study, the electron beam was misaligned purposefully. The bunch, now passing through the highly nonlinear beam edge, quickly gained emittance and lost particles.
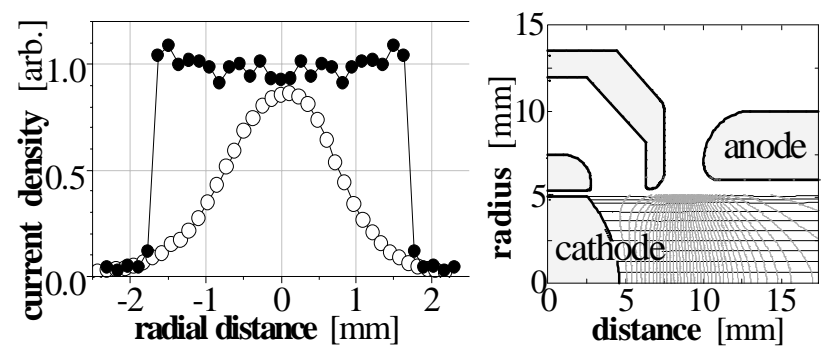
This unfortunate effect spurred the design of a new gun with a very smooth, almost Gaussian-shaped profile. The perveance of the Gaussian gun is only $1.8 \mu \mathrm{P}$ vs 5.6 for "flat profile" gun, but the central current density is about the same than that of the "flattop" gun. Figure 6 shows transverse distribution of the electron current density generated in the "Gaussian" and "flattop" beams [5].

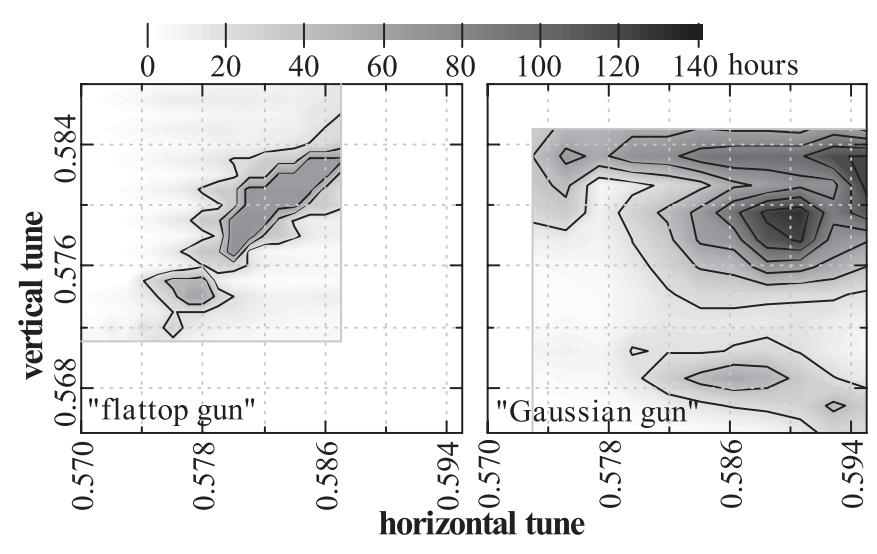

Figure 7 supplies cogent evidence that a smoother beam profile can preserve the bunch lifetime. Two workingpoint scans (measuring lifetime at various horizontal and vertical tunes) were conducted - the first with the "flattop" gun, the second with the "Gaussian" gun. While the two scans did not cover exactly the same regions of tune space, most of each scan overlaps.

The plots have identical boundaries and color scales, and contours are drawn every 20 hours. The "flattop" gun could not surpass 70 hours, and its highest lifetimes were confined to a small diagonal region. On the other hand, the "Gaussian" gun offered lifetimes exceeding 120 hours over a much broader area. Again, these values are indistinguishable from typical Tevatron lifetimes. The TEL-induced tune shift in both scans was set to about 0.004 .

\section{BBC: SUPPRESSION OF "SCALLOPS"}

The very first evidence of successful BBC was suppression of vertical emittance growth of antiproton bunches tuneshifted by the TEL.

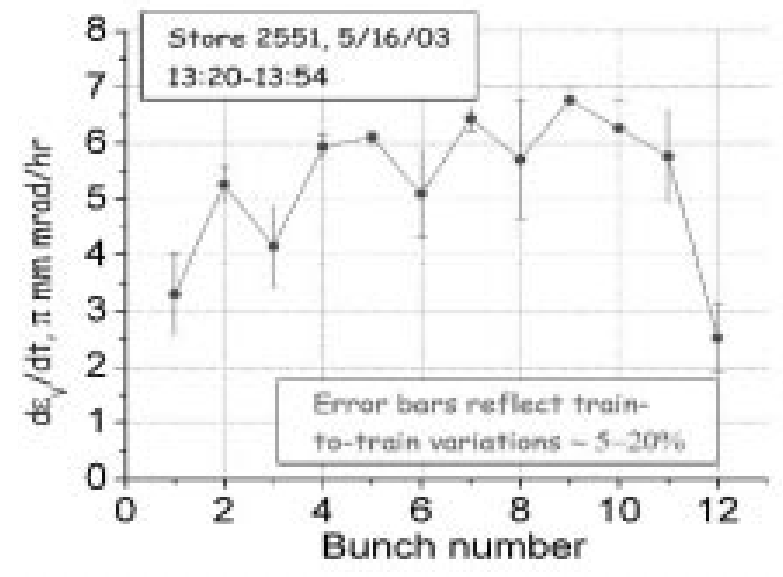

Fig.8: Emittance growth of pbar bunches

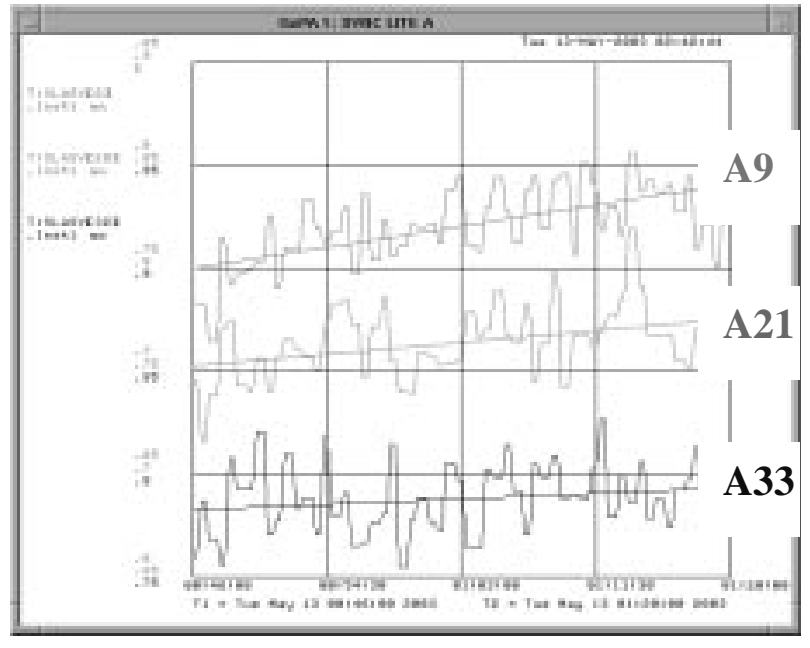

Fig.9: Vertical size of three pbar bunches in store 2540

The transverse antiproton emittance growth after 980 $\mathrm{GeV}$ beams are brought to collision is caused by beambeam interaction and occurs in the Tevatron when proton bunch intensity exceeds $180 \mathrm{e} 9$ [9]. Fig. 8 shows emittance growth rate of 12 antiproton bunches in the first 34 minutes of Tevatron HEP store \#2551. Because of 3fold symmetry of proton loading, the emittance growth rates are the same within 5-20\% for corresponding bunches in different trains (e.g. for $\# 1,13,25$ or for $\# 2,14,26$, etc) - as indicated by error bars in Fig.8. One can see that blowup rates are smaller for bunches closer to the end or start of the train. For comparison, emittances of all the bunches before collisions are very similar, in the range of $18-22 \pi \mathrm{mm} \operatorname{mrad}$ (95\% normalized). After about 1 hour the blowup flattens out, and distribution of pbar emittances over different bunches looks like three "scallops". The "scallops", though, do not appear in every store because the effect is dependent on antiproton tunes, particularly how close one of them is to some important resonance. For a typical working point of $Q_{x}=0.582$, $Q_{y}=0.590,5^{\text {th }}$ order $(0.600), 7^{\text {th }}$ order $(0.5714)$ and $12^{\text {th }}$ order (0.583) resonances play major role in the pbar beam dynamics [9]. In April-May 2003 it was observed that vertical tune changes as small as -0.002 often resulted in a reduction of the amplitude of the "scallops". Smaller but still quite definite "scallops" were also seen in protons.

The TEL was used at the beginning of several HEP stores in attempt to reduce the "scallops". First, it was demonstrated that the TEL can be transferred from DC beam removal regime to the $\mathrm{BBC}$ regime, that includes manual changing of the e-gun cathode voltage from $6 \mathrm{kV}$ to $4.5 \mathrm{kV}$ and increase of cathode filament power from $39 \mathrm{~W}$ to $46 \mathrm{~W}$ (all that in order to increase electron space charge), changing triggering from 3 pulses every $7^{\text {th }}$ turn (DC cleaning) to 1 pulse every turn (for BBC), changing electron pulse timing (from abort gap to one of bunches), shortening the e-pulse width, and, finally, using strong TEL dipole correctors to move e-beam in the interaction region by several millimeters on pbars. All these steps 
with zero electron current produced no significant effect on colliding beams or detector backgrounds, thereafter the TEL with about $0.6 \mathrm{~A}$ of current was timed on a single pbar bunch at the beginning of the Tevatron stores and we observed that the TEL can slow vertical emittance growth of the antiproton bunch it was timed on.

Fig. 9 presents evolution of vertical rms sizes of three antiproton bunches \#9, 21 and 33 over the first 34 minutes after "initiating collisions" in store \#2540 (May 13, 2003). The TEL was acting only on bunch \#33. The size has been measured with use of SyncLite Monitor [10]. Corresponding emittance growth was $4.1 \pi \mathrm{mm} \mathrm{mrad} / \mathrm{hr}$ for bunch \#9, $2.2 \pi \mathrm{mm} \mathrm{mrad} / \mathrm{hr}$ and only $1.0 \pi \mathrm{mm}$ $\mathrm{mrad} / \mathrm{hr}$ for \#33. We consider that as evidence of the improvement due to the TEL At the beam parameters: current $0.6 \mathrm{~A}$, energy $4.5 \mathrm{kV}$, rms e-beam size $0.8 \mathrm{~mm}$, interaction region length $2.05 \mathrm{~m}$ - expected maximum horizontal pbar tune shift was about -(0.003-0.004), vertical -0.001 (estimated). After 34 minutes the TEL was turned off, and emittances of all three bunches leveled.

During 4 weeks in April-May there were 8 attempts to do the BBC at the beginning of the HEP stores. There were no "scallops" in three stores \#2445, 2490, 2495 and though the TEL was acting on antiprotons we observed no effect on emittance growth, as well as pbar losses and lifetime. Only Schottky power detector channel SHPWR responded to the TEL current by $0.5 \mathrm{~dB}$ rise. Faulty TEL pulse generator led to emittance excitation by noise and quick (1 min) loss of corresponding antiproton bunches in stores \#2487 and \#2502 - but did not lead to loss of stores. After that was fixed, we had "scallops" and the TEL on bunch \#33 in three stores and we suppressed the vertical emittance growth in \#2540, effect was neutral in \#2546, and somewhat negative (faster emittance growth) in \#2549. The Table below summarizes emittance growth rates for three "equivalent" pbar bunches (namely, bunches \#9 in each of three trains) in 3 stores with TEL off and three stores with TEL on bunch \#33 only.

\begin{tabular}{l|llll}
\hline store & duration & $A 9$ & A21 & A33 \\
\hline 2536 & $40 \mathrm{~min}$ & 9.9 & 9.2 & 9.3 \\
2538 & $35 \mathrm{~min}$ & 1.9 & 1.7 & 2.8 \\
$\mathbf{2 5 4 0}$ & $34 \mathrm{~min}$ & 4.1 & 2.2 & $\mathbf{1 . 0}$ \\
$\mathbf{2 5 4 6}$ & $30 \mathrm{~min}$ & 3.9 & 1.9 & $\mathbf{4 . 0}$ \\
$\mathbf{2 5 4 9}$ & $26 \mathrm{~min}$ & 4.5 & 3.6 & $\mathbf{7 . 1}$ \\
2551 & 34min & 6.7 & 6.6 & 7.0 \\
\hline
\end{tabular}

One can see that without the TEL, emittance growth rates over the first 30-40 minutes of the stores for the three "equivalent" bunches were the same.

Again, the effect of the TEL is obvious, though not well controlled as it can be negative as well as positive. The uncertainty is - as we think - due to insufficiently precise centering of electron beam on antiprotons. Pbar orbit at F48 can migrate by upto $0.5 \mathrm{~mm}$ over a time scale of 12 hours and upto $1 \mathrm{~mm}$ over a scale of few days to a week [11]. Unfortunately, electrical centers of the TEL BPMs are dependent on the signal bandwidth, and the difference between short pbar pulse position and long electron pulse position can not be determined with accuracy better than
0.5-1.5 mm (though, resolution of the BPMs for any of the beams alone is about 20-40 microns). Such errors in positioning of $\sigma=0.8 \mathrm{~mm}$ electron beam wrt $\sigma=0.5 \mathrm{~mm}$ pbar bunch may result, for example, in significant variation of the TEL-induced tuneshift and even in changing sign of the tuneshift. We plan to improve the TEL BPMs [8].

\section{CONCLUSIONS, FUTURE STEPS}

For the past 18 months, the TEL has been needed to clean the abort gap of residual particles. Recently we have got indications that the TEL can compensate beambeam effects in the Tevatron - it reduces "scallops".

We plan to continue experimental studies of the BeamBeam Compensation with the TEL at F48 which can be used not only for suppression of the "scallops" but also at the other stages of the Tevatron cycle (at injection energy, ramp, squeeze, during collisions). We may want to act on protons as well to do BBC or suppress coherent instabilities.

We will study effects of coherent longitudinal [12] and transverse waves in electron-(anti)proton interaction and explore the need of a better high frequency stabilization of the electron current and position.

We plan to improve the TEL BPMs and commission bunch-by-bunch tune diagnostics with $1.7 \mathrm{GHz}$ Schottky detector [13].

Fabrication of the second electron lens in collaboration of IHEP (Protvino) is underway and will be finished in the summer of 2004.

Possible hardware changes are focused on having wider electron beam with higher current in the TEL and include : a) add solenoidal coils in the bends of the TEL in oder to allow propagation of electron lens with smaller field in the main superconducting solenoid; b) new $15 \mathrm{kV} \mathrm{HV}$ modulator; c) new electron gun combining flattop and smooth "Gaussian" tails.

The number of people involved with the TEL has evolved and increased over the past few years. Appreciation goes to H.Pfeffer, G.Saewert, A. Semenov, D. Wildman, D. Wolff, and M. Olson, all of who have contributed considerable effort.

\section{REFERENCES}

[1] V.Shiltsev, D.Finley, FNAL-TM-2008 (1997)

[2] V.Shiltsev, FNAL-TM-2031 (1997)

[3] V.Shiltsev, et.al, Phys.Rev. ST-AB, 2,071001(1999)

[4] D.Shatilov, et.al, Proc.PAC'01, p.2002

[5] Y.Alexahin, et.al, Proc.PAC'01, p.2005

[6] V.Shiltsev, et.al, Proc.PAC'01, p.158

[7] K.Bishofberger, et.al, Proc. Advanced Accelerator

Concepts Workshop '02, p. 821.

[8] X.Zhang, et.al, Proc. PAC'03.

[9] T.Sen, X.Zhang, et.al, Proc. PAC'03.

[10] H.Cheung, et.al, Proc. PAC'03.

[11] V.Shiltsev, et.al, FNAL-Conf-02/250 (2002).

[12] V.Parkhomchuk, et.al, .Zh.Tech.Phys, 73,8 (2003)

[13] R.Pasquinelli, Proc. PAC'03. 
